5-1-1992

\title{
Optical Caustics in Natural Phenomena
}

James A. Lock

Cleveland State University, j.lock@csuohio.edu

James $\mathrm{H}$. Andrews

Case Western Reserve University

Follow this and additional works at: https://engagedscholarship.csuohio.edu/sciphysics_facpub

Part of the Physics Commons

How does access to this work benefit you? Let us know!

\section{Publisher's Statement}

Copyright 1992 American Association of Physics Teachers. The article appeared in American Journal of Physics 60 (1992): 397-407 and may be found at http://aapt.scitation.org/doi/

$10.1119 / 1.16891$

Original Citation

Lock, James A. and J. H. Andrews. "Optical Caustics in Natural Phenomena." American Journal of Physics 60 (1992): 397-407.

\section{Repository Citation}

Lock, James A. and Andrews, James H., "Optical Caustics in Natural Phenomena" (1992). Physics Faculty Publications. 83.

https://engagedscholarship.csuohio.edu/sciphysics_facpub/83

This Article is brought to you for free and open access by the Physics Department at EngagedScholarship@CSU. It has been accepted for inclusion in Physics Faculty Publications by an authorized administrator of

EngagedScholarship@CSU. For more information, please contact library.es@csuohio.edu. 


\title{
Optical caustics in natural phenomena
}

James A. Lock

James $\mathrm{H}$. Andrews

\begin{abstract}
When observing a distant point source of light through a water droplet adhering to a pane of glass near one's eye or the scattering of light from raindrops, one often sees optical caustics. In this paper, diffraction integrals are used to investigate these caustics. The caustic shapes are related to divergences in the stationary phase method for approximating the diffraction integrals. These divergences correspond to the coalescing of two or more geometrical light rays in ray optics or the coalescing of two or more regions of stationary phase in wave optics. The number of coalescing light rays is related to a polynomial approximation of the phase function in the diffraction integral. Also, the relation between the shape of the resulting caustic and the elementary caustic forms of the catastrophe optics classification scheme is described.
\end{abstract}

\section{INTRODUCTION}

If at night you look at a distant street lamp through a drop of water on your eyeglasses or on a window pane close to your eye, you will see not just the image of the street lamp, but also a webbing of bright light surrounded by interference patterns of lesser brightness. ${ }^{1}$ Such a webbing is shown in Fig. 1. Similarly, a gentle late afternoon rain occasionally reveals the bright arc of a rainbow and sometimes the supernumerary interference pattern adjacent to it. ${ }^{2}$ The curving bright lines in these natural phenomena are examples of optical caustics. Postponing for the moment a precise definition of an optical caustic, one can say that it is associated with some degree of focusing of reflected or refracted light. The purpose of this paper is to understand how the optical caustics in these phenomena are formed. In doing so, we describe a convenient method for both characterizing and quantitatively describing them. Neither the analyses of the natural phenomena discussed in this paper nor the method for characterizing caustics that we describe is fundamentally new. But much of the literature on optical caustics employs rather specialized vocabularies that may be somewhat foreign to an interested but uninitiated reader. In this paper we demonstrate that a description of optical caustics based on wave interference is straightforward and, at the introductory level, does not require the specialized vocabularies used in much of the current research on optical caustics.

Optical caustics may be described either in terms of light rays or in terms of light waves. A simple example suffices to explain what an optical caustic is and how its ray optics and wave optics descriptions are related. Consider a perfectly focusing circular lens of diameter $d$ and focal length $f$. If a family of parallel light rays is incident along the symmetry axis of the lens, all of the refracted rays pass through the focal point producing an infinite light intensity there. The focal point is an optical caustic. In fact, the locus of all the points at which the intensity of the light rays that are reflected or refracted by an object is infinite is one of the definitions of an optical caustic.

Ray optics is valid, however, only when the sizes of all the pertinent objects and structures in the problem are much larger than the wavelength $\lambda$ of the light that is used. This criterion is violated at the infinitesimally small focal point of the perfectly focusing lens, and at every point on an optical caustic in general. Thus the intensity of the light in the vicinity of a caustic is more properly calculated using wave optics. For example, it is relatively easy to show with wave optics that the infinite intensity focal point of a perfect lens becomes a focal waist of large but finite intensity whose cross section in the focal plane is the central maximum of the circular aperture Fraunhofer diffraction pat$\operatorname{tern}^{3}$

$$
I(r) \propto\left(\frac{J_{1}(\pi r d / \lambda f)}{(\pi r d / \lambda f)}\right)^{2},
$$

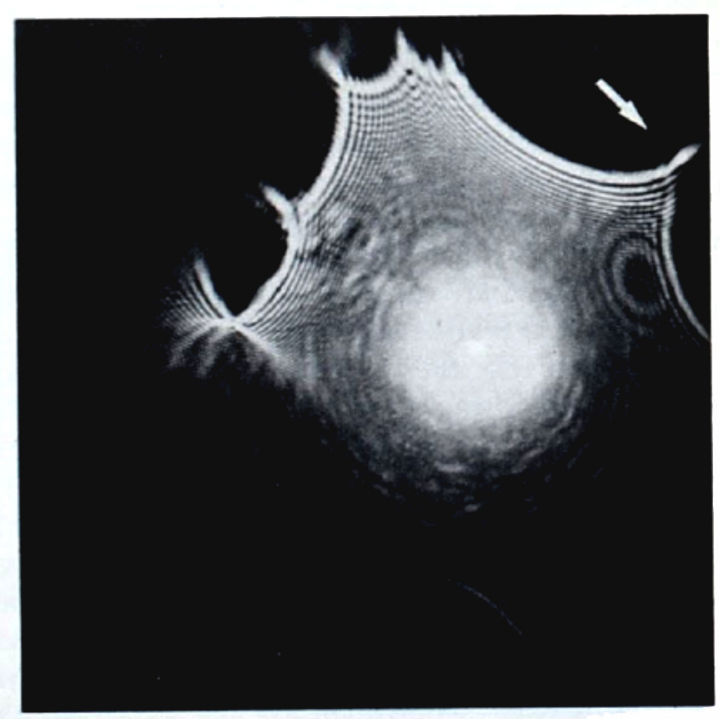

Fig. 1. Photograph of far-field caustic of a water droplet adhering to a vertical glass microscope slide and illuminated by an expanded beam of a $\mathrm{He}-\mathrm{Ne}$ laser. The arrow marks one of the prominent cusps produced by irregularities near the edge of the droplet. 
where $r$ is the distance in the focal plane from the geometrical focal point. The central maximum, sometimes referred to as the Airy disk, is surrounded by the remainder of the diffraction pattern of Eq. (1). A way from the focal plane in either direction, the intensity is a circular aperture Fresnel diffraction pattern.

The example of a perfectly focusing lens can also be used to motivate a qualitative characterization of two different approaches that have been taken in analyzing optical caustics. One approach can be said to emphasize the focusing properties of the lens, and the other emphasizes the diffraction pattern in the vicinity of the caustic. In the first approach, if one considers a real lens rather than the idealization of a perfectly focusing one, the ray optics caustic produced by an incident plane wave grows from a single focal point to a more complicated three-dimensional structure due to the aberrations of the lens. Points on the caustic may correspond to the focusing of an infinite number of light rays due to the rotational symmetry of the circular lens. The various lens aberrations and the caustics that they produce have been described in great detail in terms of both the refraction of light rays through the lens and the changes in the curvature of the exiting wave fronts produced by the lens. These methods give the exact shape of the entire caustic produced by the reflecting or refracting surface, and greatly simplify for high symmetry situations such as a lens or a system of lenses. ${ }^{4 \rightarrow}$

The second approach is appropriate for the caustics produced by plane waves incident on various reflecting or refracting surfaces that do not possess the high rotational symmetry of a circular lens. For these surfaces, only a small number of light rays focus to form the caustic. The electric field in the vicinity of these caustics can be written as an integral over the phase $\Phi$ associated with each of the optical paths that light waves can take in going from the reflecting or refracting surface to an observation point. ${ }^{8-12}$ These integrals are known as diffraction integrals and have been discussed in an intuitive way in a popular book by Feynman. ${ }^{13}$ Consider a diffraction integral of the form

$$
E\left(x_{0}, y_{0}, z_{0}\right)=\iint e^{i \phi\left(x, y, x_{0}, y_{0}, z_{0}\right)} d x d y,
$$

where the $x y$ plane is the entrance plane of the irregular reflecting or refracting surface and $\left(x_{0}, y_{0}, z_{0}\right)$ are the coordinates of an observation point. The value of the integral is dominated by the small number of regions in the $x y$ plane of integration where the phase function $\Phi$ is stationary. These correspond to regions of constructive interference of the light waves and are identified ${ }^{13}$ with the geometrical light rays that form the caustic and its surrounding interference structure. For the remainder of the $x y$ plane, the phase function $\Phi$ is rapidly varying and corresponds to destructive interference. It contributes little to the value of the integral in Eq. (2).

The purpose of the next two sections of this paper is to show that a simple and convenient way to describe the optical caustics observed in the natural phenomena described at the beginning of this section is in terms of a diffraction integral such as Eq. (2) where the phase function $\Phi$ has been approximated by a polynomial of low degree in $x$ and $y$. As we will show, the degree of the polynomial in $x$ and $y$ is related to the maximum number light rays which can focus to form the caustic. This is the second approach referred to above. It cannot be applied to situations involving circular symmetry because an infinite number of rays focus to form the caustic. The first approach, used for lens systems and high symmetry situations, can be applied to low symmetry irregular reflecting or refracting surfaces and gives the exact shape of the resulting caustic. The calculations involved in doing so, however, become quite complicated. The polynomial approximation to $\Phi$ in Eq. (2), on the other hand, implies that all the details of the caustic calculated in this way are not rendered exactly. But enough terms in the Taylor series expansion of $\Phi$ are retained to preserve all of the caustic's fundamental st ructure and features. Thus the polynomial approximation of $\Phi$ permits a great simplification in the calculation of the shape of the caustic and the interference structure that surrounds it when the caustic is due to the focusing of only a few rays.

Since the caustics described at the beginning of this section are produced by two different mechanisms acting on two different types of water droplets, i.e., refraction through an irregularly shaped droplet and scattering by a spherical droplet, the derivations of the forms of the diffraction integrals describing each phenomenon in practice proceed in different ways. We consider scattering by a spherical droplet in Sec. II and refraction through an irregularly shaped droplet in Sec. III. In Sec. II we derive the applicability of diffraction integrals and polynomial approximations to the phase functions from basic principles in the context of the simplest caustic, the rainbow. In Sec. III we demonstrate the power and simplicity of the use of polynomial approximations to the phase function for more complicated caustics.

\section{CAUSTICS AND LIGHT SCATTERING}

One can describe the rainbow appearing in the far-field scattering of light by a spherical water droplet using either ray optics or wave optics. We briefly review first the ray optics treatment and then the Airy theory of the rainbow in order both to motivate our wave optics analysis and to introduce our notation. In ray optics, a family of parallel rays is incident on a water droplet of radius $a$ and refractive index $n$. Each ray, as in Fig. 2, has a different impact parameter $b$ with respect to the droplet center, and the angle of incidence of such a ray with the droplet surface is $\theta$, where

$$
\sin \theta_{i}=b / a \text {. }
$$

The ray incident with the angle $\theta$, is deflected through the angle $\Theta$ during the scattering process. If the scattering process is identified with transmission into the droplet, one internal reflection, and then transmission out of the droplet, ${ }^{14}$ the deflection is given by

$$
\Theta\left(\theta_{i}\right)=\pi+2 \theta_{i}-4 \theta_{,},
$$

where

$$
\sin \theta_{i}=n \sin \theta_{\text {. }}
$$

Since $\Theta \leqslant \pi$ for this process, the deflection angle is identical to the scattering angle $\theta$. For other scattering processes, such as transmission out of the droplet after two or more internal reflections, the deflection angle of the emerging ray is greater than $\pi$. For those processes, the relation between the deflection angle and the scattering angle, which is defined to be less than or equal to $\pi$, is somewhat more complicated.

By relating the power incident on the portion of the droplet surface bounded by the angles $\theta_{i}$ and $\theta_{i}+d \theta_{i}$ to 


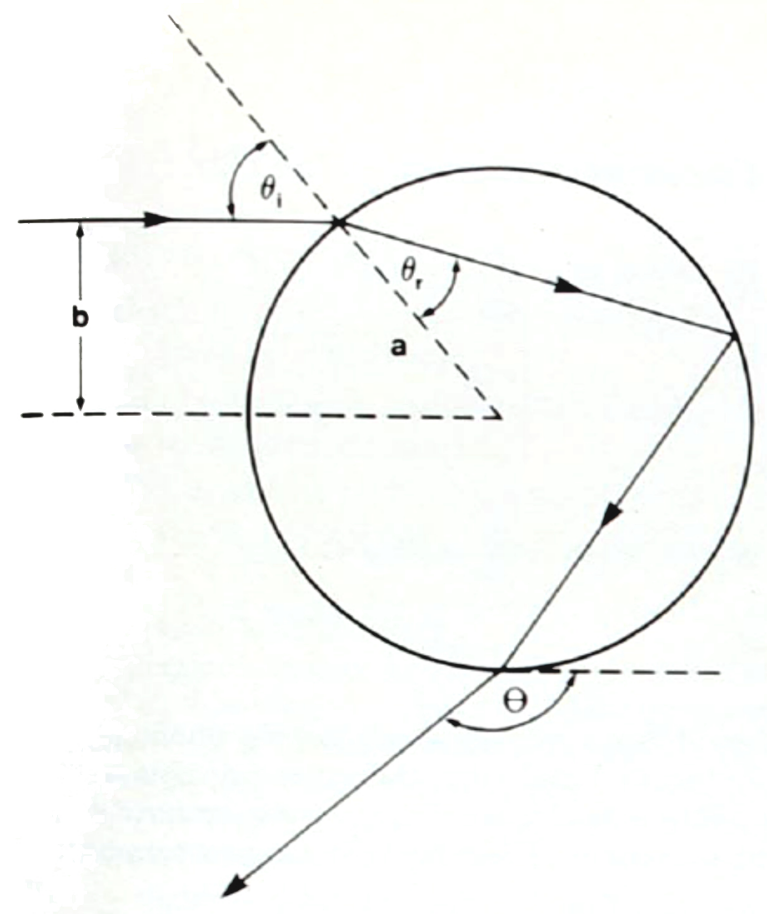

Fig. 2. Schematic of a water droplet showing an internally reflected light ray incident at the angle $\theta_{i}$. The ray has the impact parameter $b$ and it is scattered through the angle $\Theta=\theta$.

the scattered power entering the far field and bounded by the angles $\Theta$ and $\Theta+d \Theta$, the scattered intensity is found to be $^{14}$

$$
\begin{aligned}
I(\Theta)= & \frac{I_{0} a^{2}}{R^{2}} \frac{\sin \theta_{i} \cos \theta_{i}}{\sin \Theta} T_{21}\left(\theta_{i}\right) R_{11}\left(\theta_{i}\right) T_{12}\left(\theta_{i}\right) \\
& \times \frac{1}{\left|d \Theta / d \theta_{i}\right|} .
\end{aligned}
$$

In this expression, $I_{0}$ is the incident light intensity, $R$ is the distance from the water droplet to the observer, $T_{21}$ and $T_{12}$ are the fractions of the intensity (with a given incident polarization state) transmitted into and out of the droplet, respectively, and $R_{11}$ is the fraction of the intensity that is internally reflected. ${ }^{15}$ For unpolarized incident light, the parallel and perpendicular polarization states must be treated separately and the resulting intensities are then summed.

The deflection angle of Eq. (4) possesses a single relative minimum at the incident angle $\theta_{i}^{D}$ given by

$$
\cos ^{2} \theta_{i}^{D}=\left(n^{2}-1\right) / 3 \text {. }
$$

The ray incident at this angle is known as the Descartes ray, or the rainbow ray. For this ray, $d \Theta / d \theta_{i}$ vanishes and $I(\Theta)$ diverges signaling the presence of a caustic. The corresponding scattering angle

$$
\theta^{D}=\Theta\left(\theta_{i}^{D}\right)
$$

is known as the rainbow scattering angle.

An improvement upon ray theory is provided by the Airy theory of the rainbow. Airy theory is in a sense a combination of the ray theory approach to caustics mentioned in Sec. I and the diffraction integral approach of Sec.
III. In the first step of Airy theory, one follows the paths of the geometrical rays through the water droplet and determines that an initially flat wave front exits the droplet in the vicinity of the rainbow scattering angle with a cubic shape. ${ }^{16}$ In the second step of the theory, the cubic exiting wave front is Fraunhofer diffracted into the far field. The Fraunhofer diffraction integral is of the form of Eq. (2) and is recognized as the Airy integral. ${ }^{17,18}$

The purpose of the remainder of this section is to show from first principles that the rainbow caustic appearing in the far-field light scattering by a spherical water droplet (and by analogy the more complicated caustics described in the next section as well) may be described by a diffraction integral. The derivation of a similar diffraction integral for quantum mechanical scattering by a spherically symmetric potential whose range is much larger than the de Broglie wavelength $\lambda$ is known as the semiclassical approximation to scattering and was carried out by Ford and Wheeler many years ago. ${ }^{19}$ The derivation of the diffraction integral for electromagnetic scattering by a spherical water droplet (the rainbow caustic) proceeds in an almost identical manner. In this derivation we employ the vocabulary of scattering theory and point out analogies between quantum mechanical and electromagnetic scattering. A number of the results we find for the resulting one-dimensional diffraction integral will be repeated and generalized to two dimensions as we examine the more complicated caustics of the next section. Readers primarily interested in the description of caustics and their associated wave fields or who are familiar with the scattering theory motivation for diffraction integrals may turn to the last paragraph of this section without any loss of continuity.

We begin our derivation of the diffraction integral for the rainbow caustic with an exact solution to the electromagnetic boundary value problem of a plane electromagnetic wave incident on a dielectric sphere. In the simpler boundary value problem provided by quantum mechanical scattering, when the incident and scattered wave functions are decomposed into sums of partial waves, the far-field scattered wave function takes the form

$$
\psi_{\text {scattered }}(R, \theta, \phi)=\left(e^{i k R} / k R\right) F(\theta),
$$

where the scattering amplitude $F$ is given by

$$
F(\theta)=\sum_{l=0}^{\infty}(2 l+1) f_{l} P_{l}(\cos \theta) .
$$

The coefficients $f_{l}$ are called the partial wave scattering amplitudes. In electromagnetic scattering, the incident plane wave, and the internal and scattered electric and magnetic fields are also decomposed into sums of partial waves. The partial waves are the transverse electric (TE) and transverse magnetic (TM) spherical multipole waves familiar from radiation and antenna problems. ${ }^{20}$ If the incident electric field has strength $E_{0}$ and is polarized in the $\hat{u}_{x}$ direction, then matching the boundary conditions for the various components of the electric and magnetic fields at the surface of the water droplet gives an exact solution to the electromagnetic boundary value problem known as the Mie scattering solution. One has in the far field ${ }^{18,21,22}$

$$
\begin{aligned}
\mathbf{E}_{\text {scattered }}(R, \theta, \phi)= & i E_{0}\left(e^{i k R} / k R\right) \\
& \times\left[S_{1}(\theta) \sin \phi \hat{u}_{\phi}+S_{2}(\theta) \cos \phi \hat{u}_{\theta}\right],
\end{aligned}
$$


where the electromagnetic scattering amplitudes $S_{1}$ and $S_{2}$ are given by

$$
S_{1}(\theta)=\sum_{l=1}^{\infty} \frac{2 l+1}{l(l+1)}\left[a_{l} \pi_{l}(\theta)+b_{l} \tau_{l}(\theta)\right]
$$

and

$$
S_{2}(\theta)=\sum_{l=1}^{\infty} \frac{2 l+1}{l(l+1)}\left[a_{l} \tau_{l}(\theta)+b_{l} \pi_{l}(\theta)\right],
$$

and where the angular functions $\pi_{l}$ and $\tau_{l}$ are given by

$$
\pi_{l}(\theta)=(1 / \sin \theta) P_{i}(\cos \theta)
$$

and

$$
\tau_{l}(\theta)=\frac{d}{d \theta} P_{l}^{\prime}(\cos \theta) .
$$

The coefficients $a_{l}$ and $b_{l}$ are the partial wave scattering amplitudes for the TM and TE multipoles, respectively. They are functions of $n$ and the size parameter of the water droplet

$$
X \equiv 2 \pi a / \lambda \text {. }
$$

Their explicit forms are analogous to the explicit form of

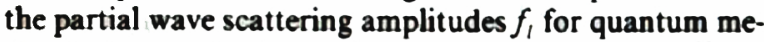
chanical scattering by a spherical straight-edge well. ${ }^{23}$

If a plane wave is incident at an angle $\boldsymbol{\theta}_{i}$ on a flat interface with either TE or TM polarization, the portions of the incident electric field that are transmitted or reflected are given by the Fresnel coefficients $t\left(\theta_{1}\right)$ and $r\left(\theta_{i}\right) .^{15}$ If the plane wave were instead incident at an angle $\theta_{1}$ on a flat thin film, the portions of the incident electric field transmitted or reflected by the film may be written as a sum of contributions, each of which depends on the single interface Fresnel coefficients and is interpreted as specular reflection, direct transmission, or transmission following an arbitrary number of multiple internal reflections. ${ }^{24}$ Similarly, if a spherical multipole wave with either the TE or TM polarization encounters a concentric spherical interface, a portion $t_{l}$ of its amplitude is transmitted with the phase change $\xi_{1}$ and a portion $r_{l}$ is reflected with the phase change $\chi_{1} \cdot{ }^{25}$ When the multipole wave interacts with a spherical water droplet, the partial wave scattering amplitudes $a$, and $b$, may be written as sums of contributions, each of which depends on the single interface factors $t, e^{i 5}$ and $r_{1} e^{x_{1}}$ and is again interpreted as diffraction, specular reflection, direct transmission, or transmission following an arbitrary number of multiple internal reflections. ${ }^{26}$ This is known as the Debye series and it allows one to decompose the total scattering amplitude into its constituent physical processes. Employing this decomposition, the portion of the partial wave scattering amplitudes that corresponds to transmission into the droplet, one internal reflection, and then transmission back out is ${ }^{27}$

$$
\begin{aligned}
& a_{i}^{1} \text { reflection }=\left[\left(t_{i}^{\mathrm{TM}}\right)^{2} / n\right]\left(r_{i}^{\mathrm{TM}}\right) e^{i\left(2 \xi i^{\mathrm{TM}}+x_{i}^{\mathrm{TM}}\right)}, \\
& \boldsymbol{b}_{i}^{\text {reflection }}=\left[\left(t_{i}^{\mathrm{TE}}\right)^{2} / n\right]\left(r_{i}^{\mathrm{TE}}\right) e^{i\left(2 \xi \xi^{\mathrm{TE}}+x_{l}^{\mathrm{TE}}\right)} .
\end{aligned}
$$

For a raindrop illuminated by visible light with $a \approx 1$ $\mathrm{mm}$, thousands of partial waves make important contributions to the scattering amplitudes $S_{1}$ and $S_{2}$. For $l>1$ and $\theta$ away from 0 or $\pi$, the angular functions $\pi_{1}$ and $\tau_{1}$ may be approximated by ${ }^{27}$

$\pi_{l}(\theta) \approx \frac{1}{\sin \theta}\left(\frac{2 l}{\pi \sin \theta}\right)^{1 / 2} \sin \left[\left(l+\frac{1}{2}\right) \theta-\frac{\pi}{4}\right]$

$$
\tau_{l}(\theta) \approx l\left(\frac{2 l}{\pi \sin \theta}\right)^{1 / 2} \cos \left[\left(l+\frac{1}{2}\right) \theta-\frac{\pi}{4}\right],
$$

and the scattering amplitudes of Eqs. (12), (13) become

$$
\begin{aligned}
S_{1}^{1 \text { reflection }}(\theta)= & \sum_{l=1}^{\infty} A_{l}^{\mathrm{TE}}\left(e^{\left.i\left[2 \xi^{\mathrm{TE}}+x_{l}^{\mathrm{TT}}+1 l+1 / 2\right) \theta\right]}\right. \\
& \left.+i e^{\left.i \mid 2 \xi^{\mathrm{TE}}+\gamma_{i}^{\mathrm{TE}}-(l+1 / 2) \theta\right]}\right)
\end{aligned}
$$

and

$$
\begin{aligned}
& S_{2}^{1} \text { refection }(\theta)=\sum_{l=1}^{\infty} A_{l}^{T M}\left(e^{i\left(2 \xi t^{M}+x_{l}^{T M}+(l+1 / 2) \theta\right]}\right. \\
& \left.+i e^{i\left[25^{T M}+x^{T M}-(l+1 / 2) \theta\right]}\right),
\end{aligned}
$$

where $A_{I}^{\mathrm{TE}}$ and $A_{1}^{\mathrm{TM}}$ are slowly varying amplitude factors.

In the $X \gg 1$ limit, the localization principle in optics ${ }^{28}$ associates a small range of partial waves centered about $l_{\text {ave }}$ with a geometrical light ray whose impact parameter is

$$
b \approx\left(l_{\text {ave }} / X\right) a \text {. }
$$

A consequence of the localization principle is that the dominant contribution to the partial wave sums of Eqs. (21), (22) is preserved if the sum over partial waves is replaced by an integral over the associated impact param. eter. ${ }^{29}$ With this replacement, we have

$$
S_{1}^{1 \text { reflection }}(\theta) \approx \int_{0}^{\infty} d l A^{\mathrm{TE}}(l)\left(e^{i \Phi_{+}^{\mathrm{TE}(l, \theta)}}+i e^{i \Phi^{\mathrm{TE}}(l, \theta)}\right)
$$

and

$$
S_{2}^{1 \text { reflection }}(\theta) \approx \int_{0}^{\infty} d l A^{\mathrm{TM}}(l)\left(e^{i \Phi_{+}^{T M}(l, \theta)}+i e^{i \phi_{-}^{I M(l, \theta)}}\right),
$$

where

$$
\Phi_{ \pm}(l, \theta) \approx 2 \xi(l)+\chi(l) \pm l \theta,
$$

for either the TE or TM polarization. The integral over $e^{i \phi}$. corresponds to rays that enter the sphere above the centerline and exit below the centerline as in Fig. 2. This will be the dominant term for scattering into the primary rainbow that we are presently calculating. The integral over $e^{-}$corresponds to rays that enter the sphere below the centerline but also exit below the centerline. This would be the dominant term, for example, for transmission following two internal reflections resulting in the second-order rainbow. Assuming that $A^{\mathrm{TE}}(l)$ and $A^{\mathrm{TM}}(l)$ are slowly varying, the scattering amplitudes of Eqs. (24), (25) are of the form of the diffraction integral of Eq. (2) except that the integration is over a single variable rather than over two. In the neighborhood of the partial wave $l^{D}$, given by Eqs. ( 3 ), (7), and (23), $A^{\mathrm{TE}}(l)$ is an order of magnitude larger than $A^{\mathrm{TM}}(l)$ due to the internal reflection of the rainbow ray occurring near the Brewster angle. This produces the strong polarization of the rainbow, and, as a consequence, we may neglect the scattering amplitude $S_{2}$ as compared to $S_{1}$.

We now discuss the evaluation of the diffraction integral of Eqs. (24), (25) in the limit $X \gg 1$. In this limit, the phase changes $\xi$ and $\chi$ have the asymptotic forms ${ }^{25}$ 


$$
\begin{aligned}
\xi^{\mathrm{TE}}(l) \approx & \xi^{\mathrm{TM}}(l) \\
\approx & \left(n^{2} X^{2}-l^{2}\right)^{1 / 2}-\left(X^{2}-l^{2}\right)^{1 / 2} \\
& -l[\arcsin (l / X)-\arcsin (l / n X)]
\end{aligned}
$$

and

$$
\begin{aligned}
\chi^{\mathrm{TE}}(l) & \approx \chi^{\mathrm{TM}}(l) \\
& \approx 2\left(n^{2} X^{2}-l^{2}\right)^{1 / 2}-l[\pi-2 \arcsin (l / n X)]-\pi
\end{aligned}
$$

independent of polarization, and the phase functions $\Phi_{+}$ for the primary rainbow become

$$
\begin{aligned}
\Phi_{ \pm}(l, \theta)= & 4\left(n^{2} X^{2}-l^{2}\right)^{1 / 2}-2\left(X^{2}-l^{2}\right)^{1 / 2} \\
& -l[\pi+2 \arcsin (l / X) \\
& -4 \arcsin (l / n X) \mp \theta]-\pi .
\end{aligned}
$$

A numerical examination of the first derivative of the function $\Phi_{-}(l, \theta)$ shows that $\Phi$ - $(l, \theta)$ has no relative maxima or minima in $l$ for any value of $\theta$ and thus contributes little to the scattering amplitude. The function $\Phi_{+}(l, \theta)$, on the other hand, is found to possess two nearby stationary points in $l$, a relative maximum and a relative minimum, for scattering angles $\theta$ somewhat larger than $\theta^{D}$. As $\theta$ approaches $\theta^{D}$, the two stationary points coalesce at the partial wave $l^{D}$. For $\theta$ less than $\theta^{D}$, the function $\Phi_{+}(l, \theta)$ has no stationary points and also contributes little to the scattering amplitude. In ray optics, for $\theta>\theta^{D}$, the two stationary points are identified with the two geometrical rays that interfere to form the supernumerary pattern below the primary rainbow in the sky. The coalescence of the stationary points as $\theta$ approaches $\theta^{D}$ is identified with the coalescing or focusing of the two supernumerary rays to form the rainbow itself. The absence of stationary points for $\theta<\theta^{D}$ is identified with the absence of light rays in Alexander's dark band above the primary rainbow in the sky. ${ }^{2}$

The locations of the stationary points of $\Phi+$ and their contributions to the scattering amplitudes may be estimated by Taylor series expanding $\Phi_{+}(l, \theta)$ about the point of coalescence $l^{D}$. But how many terms in the Taylor series must one retain in order to describe adequately the caustic that is produced? The answer is that since the coalescence of two stationary points produces the focusing at the location of the caustic, one retains the fewest number of terms in the Taylor series that exactly reproduces this pattern of coalescence of the relative maximum and minimum of the function $\Phi_{+}(l, \theta)$ of $\mathrm{Eq}$. (29). For the present situation, when $\Phi_{+}$is Taylor series expanded about $l^{D}$, retaining the terms that are at most cubic in $l-l^{D}$ produces a polynomial approximation to $\Phi_{+}$, which we call $\Phi_{+}^{\text {polynomial }}$ and for which there are also two nearly stationary points in $l$ for $\theta>\theta^{D}$ that coalesce at $l^{D}$ as $\theta$ approaches $\theta^{D}$, and that has no stationary points for $\theta<\theta^{D}$. In this approximation we have

$$
\begin{aligned}
\Phi_{+}^{\text {polynomial }}(l, \theta) \approx & \Phi_{+}\left(l^{D}, \theta\right)+X\left(\theta-\theta^{D}\right)\left(l-l^{D}\right) \\
& -X h\left[\left(l-l^{D}\right)^{3} / 3\right],
\end{aligned}
$$

where

$$
h \equiv 3 \sin \theta_{i}^{D} / 4 \cos ^{3} \theta_{i}^{D} .
$$

This cubic wave front is also the result produced by the Airy model described in the beginning of this section and is the essential element to any wave theory description of the rainbow. Equation (30) may also be recognized as the phase function of what is called the fold caustic.

For $\theta>\theta^{D}, \Phi_{+}^{\text {polynomial }}$ has stationary points at

$$
l^{S}=l^{D} \pm\left(\theta-\theta^{D}\right)^{1 / 2} / h^{1 / 2} .
$$

Substituting Eq. (30) into Eqs. (24), (25), neglecting the variation of $A(l)$ near $l^{D}$, and using the definition of the Airy integral, ${ }^{17}$ we obtain

$$
S_{1}^{1} \text { reflection }(\theta) \propto \operatorname{Ai}\left[\left(-X^{2 / 3} / h^{1 / 3}\right)\left(\theta-\theta^{D}\right)\right] .
$$

For negative values of its argument, the Airy integral is oscillatory with a slowly decreasing amplitude giving the supernumerary interference pattern. ${ }^{30}$ For positive values of its argument, it rapidly decreases monotonically giving Alexander's dark band. Although ray optics predicts that the rainbow caustic will occur when $\theta=\theta^{D}$, the Airy integral reaches its largest value when its argument is about -1.01 , corresponding to a scattering angle slightly displaced from $\theta^{D}$.

The polynomial approximation to $\Phi_{+}$and the use of the Airy integral imply that Eq. (33) is not an exact evaluation of the scattering amplitude. But the approximations introduced only affect the details of the supernumerary interference pattern far from the rainbow caustic. The approximation does not affect the scattering amplitude in the vicinity of the caustic itself. This is shown in Fig. 3. The physical basis of the approximation may be understood in the following way. The stationary points of Eq. (32) increase above and decrease below $l^{D}$ at equal rates as $\theta$ is increased beyond $\theta^{D}$. For the exact phase function $\Phi_{+}$of Eq. (29), however, the rate of increase of the $l^{S}>l^{D}$ stationary point soon lags behind the rate of decrease of the $l^{S}<l^{D}$ stationary point since it is identified with the geometrical light ray incident near the edge of the droplet which is constrained to have the impact parameter $b \leqslant a$. The $l^{S}<l^{D}$ stationary point is identified with the light ray incident nearer the center of the droplet whose impact parameter is not similarly constrained. This asymmetry, along with the variation in $A(l)$ near $l^{D}$, limits the quantitative validity of Eq.

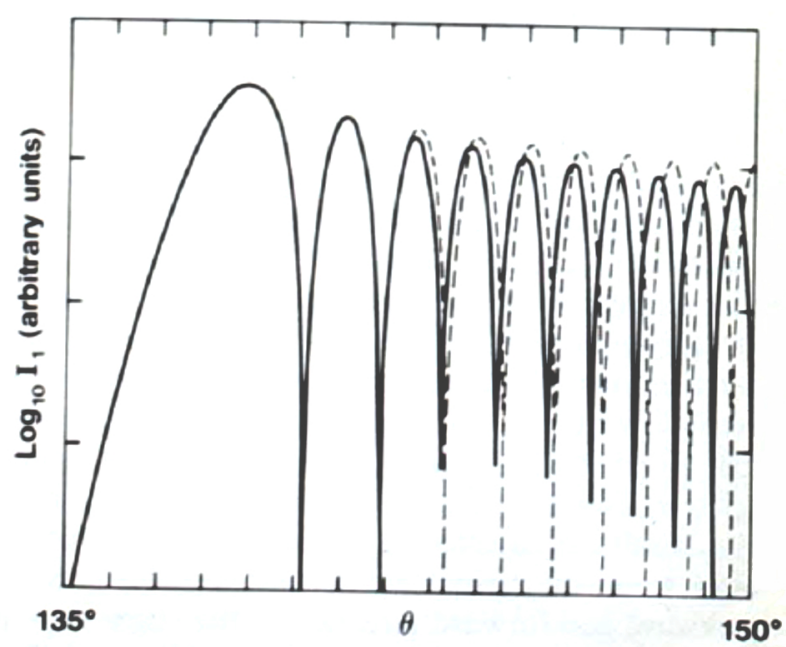

Fig. 3. Comparison between the square of the Airy integral approximation of Eq. (33) (dashed curve) and the exact evaluation (solid curve) of the scattered intensity $\mid S_{1}^{1}$ reflection $\left.\right|^{2}$ for the size parameter $X=1000$ and the refractive index $n=1.333$. 
(33) to scattering angles near $\theta^{D}$. If terms beyond third degree were considered in the Taylor series expansion of $\Phi_{+}(l, \theta)$, they would preserve the stationary point coalescence behavior of the cubic approximation of Eq. (30). They would only lessen the rate of increase of the $l^{S}>l^{D}$ stationary point with respect to the rate of decrease of the $l^{S}<l^{D}$ stationary point as $\theta$ increases beyond $\theta^{D}$. All of the fundamental physics concerning the caustic is already present in the third degree polynomial approximation of Eq. (30). Only the details of the interference pattern that surrounds the caustic are improved by the addition of higher degree terms in the Taylor series expansion of $\Phi_{+}$.

Assuming that the two stationary points of $\Phi_{+}$are somewhat separated, the integral over $e^{i \phi}$. may be approximated in a different way using the method of stationary phase. In this approximation, a Taylor series expansion about each stationary point is truncated at second degree, the resulting Fresnel integrals are analytically evaluated, and the results are added together. This is difficult to carry out for Eq. (29) since the stationary points of $\Phi+$ cannot be determined analytically in closed form. We can, however, get an idea of what happens in the stationary phase

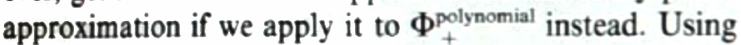
Eq. (32) for the positions of the relative maximum and minimum of Eq. (30), the stationary phase procedure gives

$$
\begin{aligned}
\operatorname{Ai}( & \left.\frac{-X^{2 / 3}}{h^{1 / 3}}\left(\theta-\theta^{D}\right)\right) \\
\propto & \frac{1}{\left(\theta-\theta^{D}\right)^{1 / 4}} \\
& \quad \times \cos \left(\frac{2}{3} \frac{X\left(\theta-\theta^{D}\right)^{3 / 2}}{h^{1 / 2}}-\frac{\pi}{4}\right) .
\end{aligned}
$$

The stationary phase approximation to the Airy integral is quite accurate when the contributing stationary points of $\Phi_{+}^{\text {polynomial }}$ are well separated for $\theta>\theta^{D}$ as is seen in Fig. 4. But as $\theta$ approaches $\theta^{D}$ and the stationary points coalesce at the rainbow scattering angle, the stationary phase approximation diverges. The rainbow caustic itself is accurately described by the integral over $e^{i \Phi}+$ and by the Airy integral approximation which is everywhere finite. But neither the original integral over $e^{i \phi+}$ nor the Airy integral may be approximated by the stationary phase method near the location of the caustic.

All of the results of this section were derived for the primary rainbow or fold caustic that occurs in far-field light scattering by a spherical water droplet. A similar analysis of the scattering amplitude can be used to derive diffraction integrals for the higher-order rainbows that occur for transmission following an arbitrary number of internal reflections. The processes of transmission out of the droplet following a certain number of internal reflections give rise to emerging geometrical rays that are in general diverging as they leave the water droplet. This diverging of the rays results in caustics appearing in the far field. An exception to the divergence of the emerging rays occurs in the backward and forward directions where light rays axially focus at infinity giving the backward or forward glory. These caustics must be calculated in a somewhat different

For light scattering by a spherical water droplet, caustic focusing also occurs after direct transmission of the light

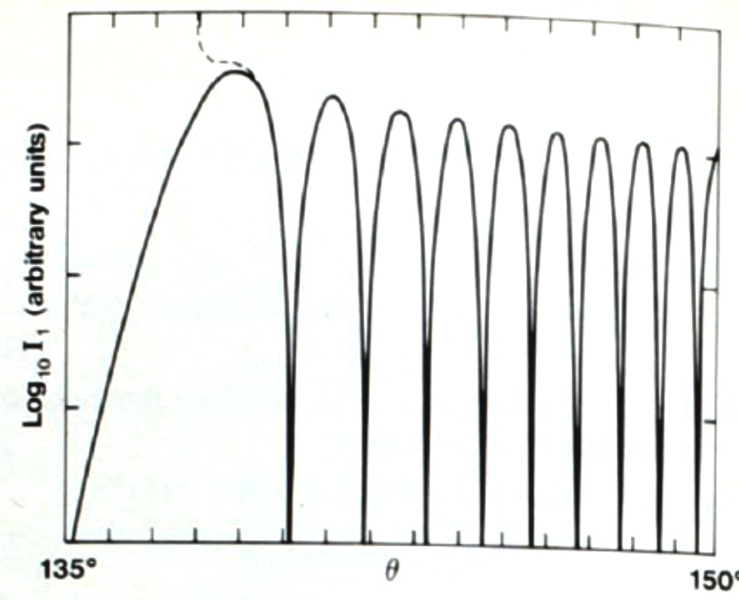

Fig. 4. Comparison between the square of the Airy integral approximation of Eq. (33) (solid curve) and the square of the stationary phase approximation of Eq. (34) (dashed curve) for the size parameter $X=1000$ and the refractive index $n=1.333$.

into and out of the droplet. In the case of direct transmission, all of the emerging light rays are converging as they leave the water droplet. This convergence produces a cuspshaped caustic of revolution accompanied by an axial caustic in the near field which are calculable using near-field scattering theory to derive the appropriate diffraction integral. ${ }^{31} \mathrm{~A}$ far simpler model that is commonly used to describe this focusing, however, is that the spherical droplet acts as a thick lens, and the shape of the near-field caustic is easily exactly calculated in ray optics using the methods of the first approach referred to in the Introduction. ${ }^{32}$

Caustics are produced in light scattering by water droplets with more complicated shapes as well. For light scattering after one internal reflection by an oblate spheroidal water droplet in the atmosphere, the emerging light rays are again diverging, and the more complicated far-field caustic that occurs ${ }^{33}$ is presumably again calculable by converting the sum over the appropriate partial wave scattering amplitudes into a diffraction integral over impact parameters as was done in this section for the simpler rainbow caustic.

Anticipating the situation treated in the next section, the electromagnetic boundary value problem for a plane wave incident on an irregular water droplet adhering to a pane of glass could in principle be solved, and a diffraction integral describing the resulting caustics could be derived from the pertinent scattering amplitudes. But, due to the low symmetry of this problem and the resulting complexity of the analysis, such a procedure has never been attempted. Rather, a much more manageable starting point related to Fermat's principle is chosen for such low symmetry problems. This starting point assumes the applicability of the diffraction integral description, rather than deriving it from more basic principles as was done in this section. This new starting point for the analysis of low symmetry situations is the topic of the next section.

\section{CAUSTICS AND DROPLETS OF WATER ON WINDOWPANES}

Consider a droplet of water on a windowpane or a pair of eyeglasses. The thickness of the droplet as a function of 
location on the glass (the $z=0$ plane) is assumed to be known and is given by $t(x, y)$. The droplet's refractive in$\operatorname{dex}$ is $\boldsymbol{n}$. The droplet is illuminated by a distant light source which we can represent by a plane wave of wavelength $\lambda$ propagating along the $z$ axis as in Fig. 5. The caustics produced by the light refracted through such droplets have been the subject of considerable study. ${ }^{11,3441}$

Our experimental observation of the caustic produced by direct transmission through the water droplet can be made either on a viewing screen placed some distance from the droplet or by looking through the droplet from very close range and focusing one's eye on the distant light source. In the former case, the electric field can be represented by Eq. (2) where the phase function $\Phi\left(x, y, x_{0}, y_{0}, z_{0}\right)$ is the optical path length of a light wave propagating from the point $(x, y)$ in the $z=0$ entrance plane of the droplet to the location $\left(x_{0}, y_{0}\right)$ on the viewing screen a distance $z_{0}$ from the entrance plane, ${ }^{42}$

$$
\begin{aligned}
\Phi\left(x, y, x_{0}, y_{0}, z_{0}\right)= & (2 \pi / \lambda)\{(n-1) t(x, y) \\
& \left.+\left[\left(x-x_{0}\right)^{2}+\left(y-y_{0}\right)^{2}+z_{0}^{2}\right]^{1 / 2}\right\} .
\end{aligned}
$$

This phase function in the paraxial approximation $\left(z_{0} \gg x, y, x_{0}, y_{0}\right)$ is given by

$$
\begin{aligned}
\Phi\left(x, y, x_{0}, y_{0}, z_{0}\right)= & \frac{2 \pi}{\lambda}\left((n-1) t(x, y)+\frac{x^{2}}{2 z_{0}}\right. \\
& +\frac{y^{2}}{2 z_{0}}-\frac{x x_{0}}{z_{0}}-\frac{y y_{0}}{z_{0}} \\
& \left.+z_{0}+\frac{x_{0}^{2}}{2 z_{0}}+\frac{y_{0}^{2}}{2 z_{0}}\right),
\end{aligned}
$$

where the last three terms may be neglected since they represent an overall phase factor that cancels out when one examines the light intensity.

If the caustic is directly observed by one's eye, the analysis proceeds similarly except that $\Phi$ is no longer a function of $z_{0}$ but of $f_{0}$, the relaxed focal length of the eye, and the electric field at the location $\left(x_{0}, y_{0}\right)$ on the retina is ${ }^{42}$

$$
E\left(x_{0}, y_{0}\right)=\iint e^{r\left(x, x_{0}, x_{0}, y_{0}\right)} d x d y,
$$

where

$$
\begin{aligned}
\Phi\left(x, y, x_{0}, y_{0}\right)= & \frac{2 \pi}{\lambda}\left((n-1) t(x, y)-\frac{x x_{0}}{f_{0}}-\frac{y y_{0}}{f_{0}}\right. \\
& \left.+f_{0}+\frac{x_{0}^{2}}{2 f_{0}}+\frac{y_{0}^{2}}{2 f_{0}}\right) .
\end{aligned}
$$

Assume that the windowpane or eyeglasses are dusty or scratched so that the water droplet's thickness $t(x, y)$ is irregular. Examination of actual water droplets shows that there are always sufficient irregularities for our purposes, especially when the supporting glass stands vertically so that the weight of the droplet is balanced by the force of its adhesion to the glass. Since the irregular water droplet does not possess circular symmetry, the caustics that it produces are formed by the focusing of only a few rays, at most $N$ of them, for example. In this section we outline a straightforward prescription for calculating the caustics produced by such droplets. We follow this prescription with a sample calculation for a realistic droplet shape.

By the method of stationary phase, the value of the electric field of Eqs. (2), (36)-(38) and thus the intensity of the refracted light at $\left(x_{0}, y_{0}, z_{0}\right)$ is dominated by the constructive interference of the light waves in the vicinity of the $N$ stationary points of $\Phi$ which we denote by $\left(x=x_{j}, y=y_{j}\right)$ or $\mathbf{r}_{j}=x_{j} \hat{u}_{x}+y_{j} \hat{u}_{y}$ for $1 \leqslant j \leqslant N$. To find these points, we need to solve

$$
\left.\nabla \Phi\right|_{r_{j}}=0,
$$

since $\left.\boldsymbol{\nabla} \boldsymbol{\Phi}\right|_{r}, \hat{u}_{r}$ is the directional derivative of $\Phi$ at $\mathbf{r}$ in the $\hat{u}$, direction. Equation ( 39 ) may be thought of as two equations in two unknowns. The unknowns are $\left(x_{j}, y_{j}\right)$, the coordinates of the geometrical light rays leaving the exit plane of the droplet on their way to the observation point $\left(x_{0}, y_{0}, z_{0}\right)$. The equations to be solved simultaneously for a given $\left(x_{0}, y_{0}, z_{0}\right)$ are

$$
\left.\left.\Phi_{x}\right|_{r_{j}} \equiv \frac{\partial \Phi}{\partial x}\right|_{r_{j}}=f_{1}\left(x_{j}, y_{j}, x_{0}, y_{0}, z_{0}\right)=0
$$

and

$$
\left.\left.\Phi_{y}\right|_{\mathbf{r}_{j}} \equiv \frac{\partial \Phi}{\partial y}\right|_{r_{j}}=f_{2}\left(x_{j}, y_{j}, x_{0}, y_{0}, z_{0}\right)=0 \text {. }
$$

Assume that we can solve these equations either analytically, numerically, or graphically to find their $\boldsymbol{N}$ solutions, the stationary points $\left\{r_{j}\right\}$. The stationary point $\mathbf{r}_{j}$ is a rela-

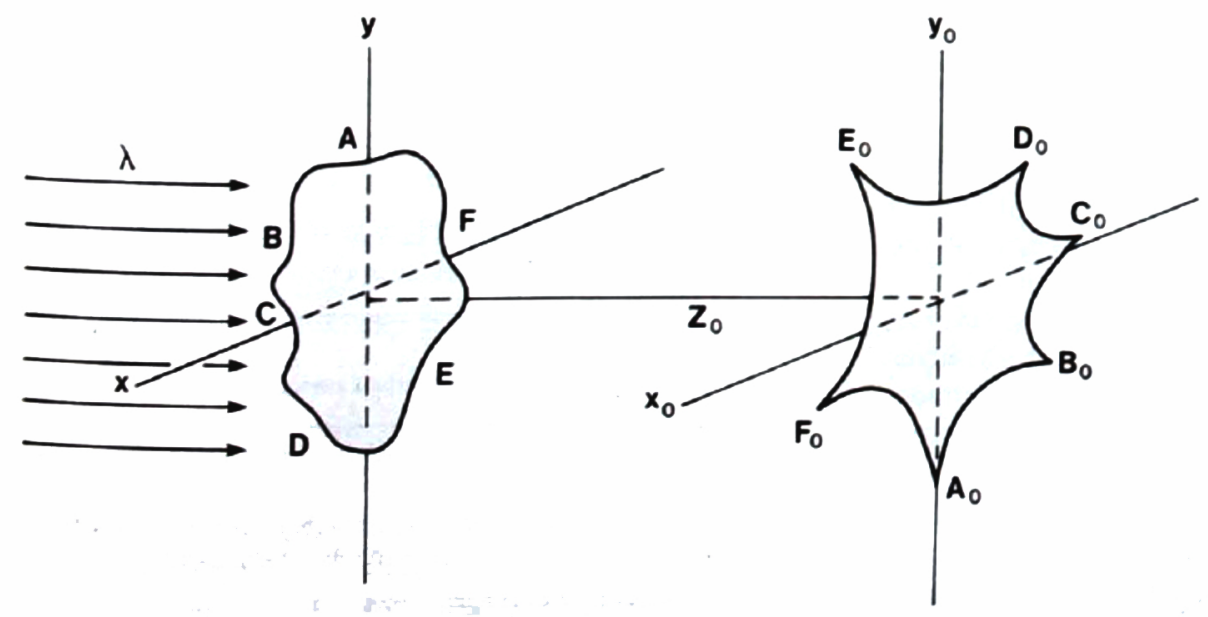

Fig. 5. Schematic of the formation of a combination of cusp caustics $A_{0}$ through $F_{0}$ by cove shaped irregularities $A$ through $F$ around the perimeter of a water droplet lens. 
tive maximum or relative minimum of $\Phi$ if $\left.\mathscr{H} \Phi\right|_{\mathbf{r}}>0$, where $^{43}$

$$
\left.\left.\left.\mathscr{H} \boldsymbol{\Phi}\right|_{\mathbf{r}_{j}} \equiv \Phi_{x x}\right|_{\mathbf{r}_{j}} \Phi_{y y}\right|_{\mathbf{r}_{j}}-\left.\Phi_{x y}^{2}\right|_{\mathbf{r}_{j}} \equiv h\left(x_{j}, y_{j}, x_{0}, y_{0}, z_{0}\right),
$$

and is a saddle point of $\Phi$ if $\left.\mathscr{H} \Phi\right|_{\mathbf{r}_{j}}<0$. Equation (42) defines the so-called Hessian of $\Phi$ evaluated at $\mathbf{r}_{j}$. It is a generalization of the second derivative for a function of two variables and tests for relative maxima, minima, or saddle points in the way that the second derivative does for a function of one variable.

If the stationary points are all separated from each other by a reasonable distance (i.e., they are isolated), we can approximate Eqs. (2), (36)-(38) very accurately using the method of stationary phase. In this method, the contribution of a stationary point to the electric field in Eqs. (2), (37) may be calculated by Taylor series expanding $\Phi$ about the point $\mathbf{r}_{j}$, neglecting terms of third order and higher, i.e., approximating

$$
\begin{aligned}
\Phi\left(x, y, x_{0}, y_{0}, z_{0}\right) \approx & \Phi\left(x_{j}, y_{j}, x_{0}, y_{0}, z_{0}\right)+\left.\frac{1}{2} \Phi_{x x}\right|_{r_{j}}\left(x-x_{j}\right)^{2} \\
& +\left.\frac{1}{2} \Phi_{y y}\right|_{r_{j}}\left(y-y_{j}\right)^{2} \\
& +\left.\Phi_{x y}\right|_{r_{j}}\left(x-x_{j}\right)\left(y-y_{j}\right)
\end{aligned}
$$

and analytically evaluating the resulting Fresnel integral. Carrying out this procedure for all of the stationary points and adding the results, we obtain

$$
E\left(x_{0}, y_{0}, z_{0}\right) \approx 2 \pi \sum_{j=1}^{N} \frac{\sigma_{j} e^{i \Phi\left(x_{j}, y_{j} x_{0}, y_{0}, z_{0}\right)}}{\left.|\mathscr{H} \Phi|_{r_{j}}\right|^{1 / 2}},
$$

where

$$
\sigma_{j}=\left\{\begin{array}{l}
+i \text { for }\left.\mathscr{H} \Phi\right|_{\mathbf{r}_{j}}>0 \text { and }\left.\Phi_{x x}\right|_{\mathbf{r}_{j}}+\left.\Phi_{y y}\right|_{\mathbf{r}_{j}}>0, \\
-i \text { for }\left.\mathscr{H} \Phi\right|_{\mathbf{r}_{j}}>0 \text { and }\left.\Phi_{x x}\right|_{r_{j}}+\left.\Phi_{y y}\right|_{\mathbf{r}_{j}}<0, \\
+1 \text { for }\left.\mathscr{H} \Phi\right|_{\mathbf{r}_{j}}<0 .
\end{array}\right.
$$

$\left.\mathscr{H} \Phi\right|_{r_{j}}$ is equal to the Gaussian curvature of the surface $\Phi(x, y)$ at the position of the stationary phase point $\mathbf{r}_{j}$.

If there is an observation point $\left(x_{0}, y_{0}, z_{0}\right)=\left(x_{0}^{c}, y_{0}^{c}, z_{0}^{c}\right)$ for which two or more of the $N$ solutions to Eqs. (40), (41) are identical, then these stationary points have coalesced and are no longer isolated. In other words, for a given observation point, if

$$
\left.\mathscr{H} \Phi\right|_{\mathbf{r}_{j}}=0
$$

then two or more stationary points of $\Phi$ coalesce in the entrance plane, producing a locally flat wave front. In ray optics this corresponds to the coalescing of two or more geometrical light rays and focusing on the viewing screen. ${ }^{12}$ The $\left.\mathscr{H} \Phi\right|_{\mathbf{r}_{j}}=0$ condition represents, for functions of two variables, a generalization of the vanishing of the second derivative of a function of one variable at the confluence of a relative maximum and minimum.

For these observation points on the caustic, the stationary phase approximation of Eq. (44) obviously is no longer applicable. In ray optics this corresponds to infinite intensity at $\left(x_{0}^{c}, y_{0}^{c}, z_{0}^{c}\right)$, and the set of all such points forms the caustic. In wave optics the electric field at the caustic is given by Eq. (2) which remains finite and is dominated by the regions of constructive interference. The classical infinity is softened by diffractive and wave interference effects.
For the irregular water droplet, how many light rays can leave the droplet at different values of $\mathbf{r}_{j}$ and pass through a single point on the viewing screen or the retina? Assuming the droplet is irregular in that it lacks the perfect circular symmetry of a spherical lens, the answer is only a few. Put another way, how many stationary points, $\mathbf{r}_{j}$, does $\Phi$ have? Again the answer is only a few. Thom's theorem of catastrophe theory ${ }^{11,12,44}$ states the surprising and significant result that if only a few rays can coalesce to form a caustic, then the coalescence can occur in only a few different ways, i.e., only a rather small number of different optical caustics, known as the stable catastrophes, can be created on the viewing screen. These caustic shapes may simultaneously occur at many locations on the viewing screen due to different irregularities at different locations on the droplet surface, and may appear in varying sizes or asymmetrically stretched, contracted and distorted. Still, there are only a few different caustic types and they can often be quickly recognized. The power of Thom's theorem is that it is not based upon the explicit form of the irregular thickness of the droplet $t(x, y)$ but only upon the maximum number of coalescing light rays it admits, that is, the maximum number of solutions that Eq. (39) has. Table I shows the connection between the number of stationary points or light rays coalescing and the number of caustics that can be so produced for up to five stationary points or rays. Pictures of these caustics appear in Refs. 11, 12, and 44.

For a given number of rays, one caustic is distinguished from another by the different ways in which all of those rays come together and coalesce to produce the strongest focusing of the caustic. Our knowledge of the shape of the droplet enables us to trace parametrically the shapes of the caustics which can be seen. In turn, the shape of the caustic can sometimes be used to identify details about the droplet's shape.

We can determine the shape of the caustic for a given $\Phi$ in the following manner. To be stationary points of $\Phi$, the coalescing points $\left(x_{j}, y_{j}\right)$ on the water droplet corresponding to the point $\left(x_{0}^{c}, y_{0}^{c}, z_{0}^{c}\right)$ on the caustic must satisfy ${ }^{45}$

$$
\begin{gathered}
f_{1}\left(x_{j}, y_{j}, x_{0}^{c}, y_{0}^{c}, z_{0}^{c}\right)=0, \\
f_{2}\left(x_{j}, y_{j}, x_{0}^{c}, y_{0}^{c}, z_{0}^{c}\right)=0, \\
h\left(x_{j}, y_{j}, x_{0}^{c}, y_{0}^{c}, z_{0}^{c}\right)=0 .
\end{gathered}
$$

For the paraxial experimental observations of Eq. (36), these three equations reduce to

$$
\begin{aligned}
& \bar{f}_{1}\left(x_{j}, y_{j}, z_{0}^{c}\right)-x_{0}^{c} / z_{0}^{c}=0, \\
& \bar{f}_{2}\left(x_{j}, y_{j}, z_{0}^{c}\right)-y_{0}^{c} / z_{0}^{c}=0, \\
& h\left(x_{j}, y_{j}, z_{0}^{c}\right)=0,
\end{aligned}
$$

Table I. Relationship between the number of coalescing rays $N$ and the number of and types of different caustics which can be produced.

\begin{tabular}{ccc}
\hline \hline$N$ & $\begin{array}{c}\text { Number } \\
\text { of caustics }\end{array}$ & Standard names of caustics \\
\hline 2 & 1 & Fold (i.e., Rainbow) \\
3 & 1 & Cusp \\
4 & 3 & Elliptic umbilic, Hyperbolic umbilic, Swallowtail \\
5 & 2 & Butterfly, Parabolic umbilic \\
\hline \hline
\end{tabular}


where $\bar{f}_{1}$ and $\bar{f}_{2}$ are the portions of the derivatives of $\Phi$ with respect to $x$ and $y$, respectively, that have no $x_{0}^{c}$ and $y_{0}^{c}$ dependence. For a given $z_{0}^{c}, h\left(x, y, z_{0}^{c}\right)=0$ defines a curve in the $x y$ plane of the droplet. Each of the points on this curve represents the location of the coalescence of at least two stationary points for some value of $\left(x_{0}^{c}, y_{0}^{c}, z_{0}^{c}\right)$. For each such point, $x_{0}^{c}$ and $y_{0}^{c}$ are given parametrically by

$$
x_{0}^{c}=z_{a}^{c} \bar{f}_{1}\left(x_{j}, y_{j}, z_{0}^{c}\right)
$$

and

$$
y_{0}^{c}=z_{a}^{c} \bar{f}_{2}\left(x_{j}, y_{j}, z_{0}^{c}\right) \text {. }
$$

If we then vary $z_{0}^{c}$ and repeat this prescription over and over again, we obtain the complete caustic in three-dimensional space.

For the paraxial experimental observations corresponding to $\mathrm{Eq}$. (38), the three equations reduce to

$$
\begin{aligned}
& \bar{f}_{1}\left(x_{j}, y_{j}\right)-x_{0}^{c} / f_{0}=0, \\
& \bar{f}_{2}\left(x_{j}, y_{j}\right)-y_{0}^{c} / f_{0}=0, \\
& h\left(x_{j}, y_{j}\right)=0 .
\end{aligned}
$$

where again $\bar{f}_{1}$ and $\bar{f}_{2}$ are the portions of the derivatives of $\Phi$ with respect to $x$ and $y$ that have no $x_{0}^{c}$ and $y_{0}^{c}$ dependence. The procedure for tracing out the shape of the caustic is similar. We are limited, however, to the single cross section of the caustic determined by $f_{0}$, the focal length of the eye, which for focusing at infinity corresponds to letting $z_{0} \rightarrow \infty$.

To simplify the evaluation of Eqs. (2), (37), instead of using the exact thickness function $t(x, y)$, we use a Taylor series expansion of it about the location in the $x y$ plane where all of the stationary points coalesce to produce the source of the strongest focusing of the caustic. If this expansion has a nonzero radius of convergence, truncating the expansion at a given degree in $x$ and $y$ provides a polynomial approximation for $\Phi$ and an approximation to the shape of the resulting caustic. Here we need to ask again, as we did in expanding the phase function for the rainbow caustic, how many terms of the Taylor series do we keep? The answer is the minimum number so that all the different coalescences of some or all of the $N$ stationary points of $\Phi$ polynomal are the same as for the $N$ stationary points of the original $\Phi$. We need not work too hard to guarantee that this will happen because, for caustics of only a few rays, only a few terms in the expansion of $\Phi$ are needed to preserve the full pattern of stationary point coalescences. ${ }^{46}$

In Table II we show the relationship between the num-

Table II. Relationship between the number of coalescing rays $\boldsymbol{N}$ and the highest degree of terms in $\Phi^{\text {pol ynomial }}$ required to produce the given caustic.

\begin{tabular}{ccc}
\hline \hline$N$ & Name of caustic & $\begin{array}{c}\text { Highest degree of terms } \\
\text { in Taylor series expansion }\end{array}$ \\
\hline 2 & Fold & Third \\
3 & Cusp & Third \\
4 & Elliptic umbilic & Third \\
4 & Hyperbolic umbilic & Third \\
4 & Swallowtail & Fifth \\
5 & Butterfly & Sixth \\
5 & Parabolic umbilic & Fourth \\
\hline \hline
\end{tabular}

ber of rays, up to five, and the degree of the polynomial phase function necessary to produce the particular caustic. By degree, we mean the combined powers of $x$ and $y$ in the polynomial. In practice, this procedure is often inverted. One does not know the exact $t(x, y)$. But by recognizing the caustic, one can work backwards to find the approximate polynomial form of $t(x, y){ }^{47}$

Replacing the phase function $\Phi$ in the diffraction integrals by a low degree polynomial in the integration variables gives only an approximate description of the interference pattern surrounding the caustic and of the shape of the caustic itself away from its strongest focusing point. The approximation, however, is quite accurate if one is close to the most strongly focusing part of the caustic, which is often the case experimentally. In this limit. Thom's description of caustics provides one with both a convenient way of classifying the types of caustics that can be produced by the focusing of a small number of light rays and a practical way of calculating the electric field strength in the vicinity of the caustic. While the stationary phase method fails at the caustic, it can still be used to calculate the diffraction structure away from the caustic.

We now apply this prescription to find parametric equations for the cusp caustic, since, for the irregular water droplet lens, the cusp is generally the most commonly encountered caustic shape. We will assume the shape of the droplet generally is a spherical cap with radius of curvature $R$. We can then approximate the thickness of the droplet in a Taylor series (neglecting terms fourth degree and higher in $x$ and $y$ ) as

$$
z=R-x^{2} / 2 R-y^{2} / 2 R \text {. }
$$

To this cap we add small irregularities in the droplet's shape due to gravity, dust, scratches on the glass, or other causes. One such irregularity might be a combination of a slight cove at the top of the droplet giving way to a slight protrusion at the bottom of the droplet. Again we expand the irregularity in a Taylor series to third degree, for example,

$$
z=\epsilon x^{2} y,
$$

where $\epsilon$ indicates the severity of the irregularity and is a positive number with dimensions of (length) ${ }^{2}$. The topography of this irregularity is shown in Fig. 6(a). Substituting Eqs. (52), (53) into Eq. ( 38$)$ as $t(x, y)$ yields

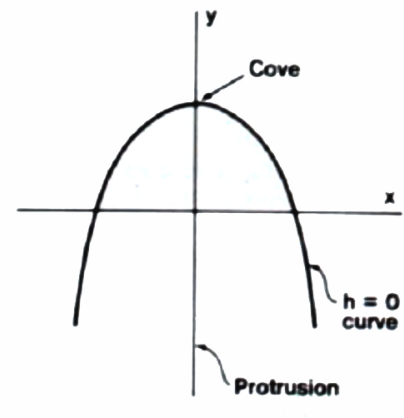

(a)

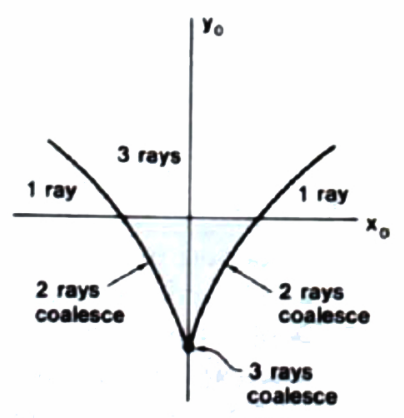

(b)
Fig. 6. (a) Schematic of the water droplet lens thickness irregularity of Eq. (53). (b) The cusp caustic of Eq. (56) resulting from the irregularity. 


$$
\begin{aligned}
\Phi^{\text {polynomial }}= & \frac{2 \pi}{\lambda}\left((n-1) R-\frac{x^{2}}{2 F}-\frac{y^{2}}{2 F}\right. \\
& \left.+\epsilon(n-1) x^{2} y-\frac{x x_{0}}{f_{0}}-\frac{y y_{0}}{f_{0}}\right),
\end{aligned}
$$

where $f_{0}$ is again the relaxed focal length of the eye and $F$ is the focal length of the spherical cap.

$$
F=R /(n-1) .
$$

Substituting Eq. (54) into Eqs. (51) and eliminating $x$ and $y$ yields the following equation for the downward pointing cusp caustic seen by the eye

$$
\left(\frac{x_{0}}{f_{0}}\right)^{2}=\frac{16}{27} F^{2} \epsilon(n-1)\left(\frac{y_{0}}{f_{0}}+\frac{1}{2 F^{2} \epsilon(n-1)}\right)^{3} .
$$

The tip of the cusp is then at

$$
\begin{aligned}
& x_{0}=0, \\
& y_{0}=-f_{0} / 2 F^{2} \epsilon(n-1),
\end{aligned}
$$

and comes from the point on the droplet

$$
\begin{aligned}
& x=0, \\
& y=1 / 2 F \epsilon(n-1) .
\end{aligned}
$$

Thus, for this thickness irregularity, the cove in the top half of the droplet produces the cusp caustic below the image of the center of the droplet. The cusp caustic of Eq. (56) is shown in Fig. 6(b). The cusp resembles the joining of two curving fold caustics at a point. Exterior to the cusp, only one light ray contributes to the electric field. Inside the cusp, three light rays contribute and produce an interference pattern that resembles the superposition of the two supernumerary patterns of the twofold caustics. In the three ray region far from the caustic, the interference pattern may be approximated using the method of stationary phase. For an arbitrary location inside the cusp, this is difficult to actually carry out since it requires solutions to cubic equations. But on the cusp's symmetry axis, the equations simplify and one obtains ${ }^{9}$

$$
I\left(x_{0}=0, y_{0}\right)= \begin{cases}I_{0}\left|y_{0} / f_{0}+1 / 2 F^{2} \epsilon(n-1)\right|^{-1}, & \text { for } y_{0}<\frac{-f_{0}}{2 F^{2} \epsilon(n-1)}, \\ I_{0}\left(y_{0} / f_{0}+1 / 2 F^{2} \epsilon(n-1)\right)^{-1}\left[3-2^{3 / 2} \sin K\left(\frac{y_{0}}{f_{0}}+\frac{1}{2 F^{2} \epsilon(n-1)}\right)^{2}\right], & \text { for } y_{0}>\frac{-f_{0}}{2 F^{2} \epsilon(n-1)} .\end{cases}
$$

where

$$
I_{0}=2 \pi^{2} / \epsilon(n-1)
$$

and

$$
K=\pi F / \lambda \text {. }
$$

Equation (59), as expected, diverges at the location of the cusp. But just as the electric field of the fold caustic is everywhere finite and is given by the Airy integral, the electric field of the cusp caustic is also everywhere finite and the diffraction integral with $\Phi^{\text {polynomal }}$ of Eq. (54) may be transformed into what is known as the Pearcey integral,, .48 i.e.,

$$
E(u, v)=\int_{-\infty}^{\infty} d t e^{u\left(t^{+}+u t^{2}+(t)\right.} .
$$

Again, if the Taylor series expansion of the cove and protrusion retained higher degree terms beyond Eq. (53), the presence of these additional terms would keep the shape of the cusp caustic near the cusp point intact. They would only perturb the shape of the caustic far from the cusp point and slightly change the details of the associated interference pattern far from the caustic.

In practice, an irregular water droplet, such as the one which produced the far-field caustic of Fig. 1, has many such coves and protrusions. If we decompose the droplet surface into various regions of influence, each cove can be thought of as the source of a cusp caustic, giving a composite caustic such as the one indicated in Fig. 5. If two adjacent coves are very close together, or if a localized region on the droplet is described by a more complicated irregularity, more complicated caustics, such as the ones cataloged in Table I, appear on the viewing screen or the observer's retina.

\section{CONCLUSIONS}

When observing the caustics that are associated with these and other natural phenomena, such as the reflection of light by warped windowpanes ${ }^{49}$ and the light refracted or reflected by the surface of a rippling stream. ${ }^{50}$ we now have a framework for understanding the caustic's shape and orientation. The caustics associated with these natural phenomena are different from the lens aberration caustics that one sometimes sees when looking at a star through a telescope in that they involve diverging rays, irregularities, or a lack of symmetry which dictates that only a small number of light rays coalesce to form the caustic. There are important and powerful ray optics methods for analyzing the exact shape of caustics generally and which may be applied to the caustics associated with natural phenomena. But, since these methods give the exact caustic shape, they are unable to capitalize on an underlying simplicity inherent when the caustic is the result of just a few coalescing rays. By calculating the caustic shapes only approximately. the underlying simplicity, i.e., the connection with catastrophe theory, becomes evident. There is not an endless variety of fundamentally different caustic forms that can be produced in systems involving only a few rays. For few-ray systems, only a few different caustic shapes can occur. Since only a small number of few-ray caustics are possible. the job of theoretically calculating them or experimentally recognizing them becomes more manageable.

\section{ACKNOWLEDGMENTS}

We thank Professor Kenneth D. Singer and Richard A. Sones for suggesting several improvements that have been incorporated in this paper. 
'M. Minnaert. The Nature of Light \& Colour in the Open Air (Dover, New York, 1954), Sec. 118

${ }^{2}$ H. M. Nussenzveig, "The theory of the rainbow,"Sci. Am. 236(4), 116-127 (1977)

${ }^{3}$ E. Hecht, Optics (Addison-Wesley, Reading. MA, 1987), second ed., p. 485.

“J. B. Keller and H. B. Keller, "Determination of reflected and transmitted fields by geometrical optics," J. Opt. Soc. Am. 40, 48-52 (1950).

${ }^{5}$ O. N. Stavroudis, The Optics of Rays, Wavefronts, and Caustics (Academic, New York, 1972).

${ }^{6}$ D. G. Burkhard and D. L. Shealy, "Flux density for ray propagation in geometrical optics," J. Opt. Soc. Am. 63, 299-304 ( 1973).

O. N. Stavroudis and R. C. Fronczek, "Caustic surfaces and the structure of the geometrical image," J. Opt. Soc. Am. 66, 795-800 (1976).

"G. B. Airy, "On the intensity of light in the neighborhood of a caustic," Trans. Camb. Phil. Soc. 6, 379-403 (1838).

${ }^{9} \mathrm{~T}$. Pearcey, "The structure of an electromagnetic field in the neighborhood of a cusp of a caustic," Phil. Mag. 37, 311-317 (1946)

${ }^{10} \mathrm{~N}$. G. Van Kampen, "The method of stationary phase and the method of Fresnel zones," Physica (Amsterdam) 24, 437-444 (1958).

"M. V. Berry, "Waves and Thom's theorem," Adv. Phys. 25, 1-26 (1976).

${ }^{12}$ M. V. Berry and C. Upstill, "Catastrophe optics: Morphologies of caustics and their diffraction patterns," Prog. Opt. 18. 257-346 (1980).

${ }^{13}$ R. P. Feynman, QED: The Strange Theory of Light and Matter (Princeton U. P., Princeton, NJ, 1985), Chap. 2.

${ }^{14} \mathrm{~J}$. D. Walker, "Multiple rainbows from single drops of water and other liquids," Am. J. Phys. 44, 421-433 (1976)

${ }^{15}$ J. R. Reitz, F. J. Milford, and R. W. Christy, Foundations of Electromagnetic Theory (Addison-Wesley, Reading, MA, 1979), third ed., Sec. 18.2 .

${ }^{16}$ W. J. Humphreys, Physics of the Air ( Dover, New York, 1964), Chap. 3.

${ }^{17}$ Handbook of Mathematical Functions, edited by M. Abramowitz and I. A. Stegun (Dover, New York, 1965), article 10.4.32.

${ }^{18} \mathrm{H}$. C. van de Hulst, Light Scattering by Small Particles (Dover, New York, 1981), Chap. 9.

${ }^{19} \mathrm{~K}$. W. Ford and J. A. Wheeler, "Semiclassical description of scattering," Ann. Phys. (NY) 7, 259-286 (1959).

${ }^{20}$ Reference 15, Sec. 17.5.

${ }^{21}$ M. Kerker, The Scattering of Light and Other Electromagnetic Radi. ation (Academic, New York, 1969), Chap. 3.

${ }^{22}$ C. F. Bohren and D. R. Huffman, Absorption and Scattering of Light by Small Particles (Wiley, New York, 1983), Chap. 4.

${ }^{23}$ E. Merzbacher, Quantum Mechanics (Wiley, New York, 1970), second ed., Sec. 11.6.

${ }^{24}$ Reference $15, \mathrm{Sec} .18 .5$.

${ }^{25} \mathrm{~J}$. A. Lock, "Cooperative effects among partial waves in Mie scattering," J. Opt. Soc. Am. A 5, 2032-2044 (1988).

${ }^{26} \mathrm{H}$. M. Nussenzveig, "High-frequency scattering by a transparent sphere. I. Direct reflection and transmission," J. Math. Phys. 10, 82124 (1969)

${ }^{27}$ Reference 18, Sec. 12.33.

${ }^{28}$ Reference 18 , Sec. 12.31 .

${ }^{29}$ This approximation in our calculation and in the calculation of Ref. 19 deletes the contributions due to what are called surface waves which turn out to be small for most scattering angles and do not influence the rainbow. Exact treatments are given in $\mathrm{B}$. van der $\mathrm{Pol}$ and $\mathrm{H}$. Bremmer, "Diffraction of electromagnetic waves from an electrical point source round a finitely conducting sphere, with applications to radiotelegraphy and the theory of the rainbow," Phil. Mag. 24, 141-176, 825-864 (1937); M. V. Berry, "Uniform approximation for potential scattering involving a rainbow," Proc. Phys. Soc. (London) 89, 479-490 (1966); H. M. Nussenzveig, "High-frequency scattering by a transparent sphere. II. Theory of the rainbow and the glory," J. Math. Phys. 10. 125-176 (1969); M. V. Berry and K. E. Mount, "Semiclassical approximations in wave mechanics," Rep. Prog. Phys. 35, 315-397 (1972); V. Khare, "Short-wavelength scattering of electromagnetic waves by a homogeneous dielectric sphere," Ph.D. dissertation, University of Rochester, 1976.

${ }^{30}$ Reference 17, Fig. 10.6

${ }^{31}$ E. A. Hovenac and J. A. Lock, private communication (1991).

${ }^{32}$ The exact shape of the caustic may be easily calculated in ray theory using the method of J. A. Lock and E. A. Hovenac, "The internal caustic structure of illuminated liquid droplets," J. Opt. Soc. Am. A 8, 15411552 (1991), appendices A and B.

${ }^{33}$ P. L. Marston and E. H. Trinh, "Hyperbolic umbilic diffraction catastrophe and rainbow scattering from spheroidal drops," Nature 312, 529-531 (1984).

${ }^{34}$ J. F. Nye, "Optical caustics in the near field from liquid drops," Proc. $\mathbf{R}$. Soc. London Ser. A 361, 21-41 (1978).

${ }^{35}$ J. F. Nye, "Optical caustics from liquid drops under gravity: Observations of the parabolic and symbolic umbilics," Phil. Trans. R. Soc. London A 292, 25-44 (1979).

${ }^{36}$ M. V. Berry, J. F. Nye, and F. J. Wright, "The elliptic umbilic diffraction catastrophe," Phil. Trans. R. Soc. London 291, 453-484 (1979).

${ }^{37}$ L. H. Tanner, "The form and motion of draining oil drops," J. Phys. D 18, 1311-1326 (1985).

${ }^{38}$ J. F. Nye, "The catastrophe optics of liquid drop lenses," Proc. R. Soc. London Ser. A 403, 1-26 (1986).

${ }^{39} \mathrm{~J}$. D. Walker, "A drop of water becomes a gateway into the world of catastrophe optics," Sci. Am. 261 (3), 176-179 (1989)

${ }^{40} \mathrm{G}$. Da Costa and R. Escalona, "Time evolution of the caustics of a laser heated liquid film," Appl. Opt. 29, 1023-1033 (1990).

${ }^{41}$ J. A. Lock, J. D. Walker, and J. H. Andrews, "Using refraction caustics to monitor evaporation of liquid drop lenses," Appl. Opt. 29, 45994607 (1990).

${ }^{42}$ J. W. Goodman, Introduction to Fourier Optics (McGraw-Hill, San Francisco, 1968 ), Sec. 4.1.

${ }^{4}$ See, for example, S. K. Stein, Calculus and Anolytic Geometry (McGraw-Hill, New York, 1987), fourth ed., Sec. 12.7.

${ }^{44}$ R. Thom, Structural Stability and Morphogenesis (Benjamin, Reading, MA, 1975).

${ }^{45} \mathrm{M}$. V. Berry, "Cusped rainbows and incoherence effects in the ripplingmirror model for particle scattering from surfaces," J. Phys. A 8, 566584 (1975).

${ }^{46}$ P. L. Marston, "Geometrical and catastrophe optics methods in scattering," in Physical Acoustics, edited by R. N. Thurston and A. D. Pierce (Academic, New York, to appear)

${ }^{47} \mathrm{H}$. Trinkhaus and F. Drepper, "On the analysis of diffraction catastrophes," J. Phys. A 10, L11-L16 (1977)

${ }^{48}$ P. L. Marston, "Transverse cusp diffraction catastrophes: Some pertinent wave fronts and a Pearcey approximation to the wave field," J. Acoust. Soc. Am. 81(2), 226-232 (1987).

${ }^{49} \mathrm{P}$. S. Theocaris, "Multicusp caustics formed from reflections of warped surfaces," Appl. Opt. 27, 780-789 (1988).

${ }^{50}$ M. V. Berry and J. F. Nye, "Fine structure in caustic junctions," Nature 267, 34-36 (1977).

Post-print standardized by MSL, Academic Endeavors, the imprint of the Michael Schwartz Library at Cleveland State University, 2017. 